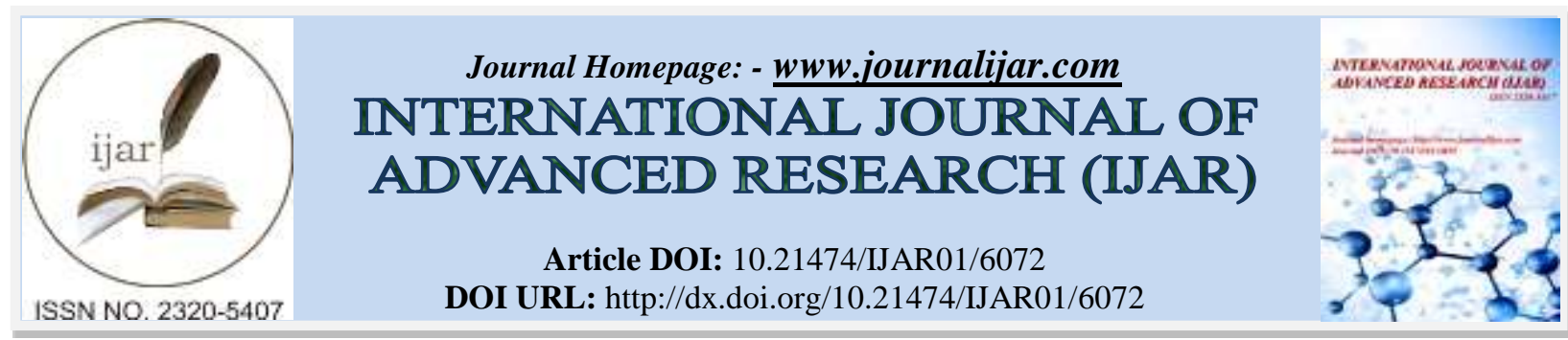

RESEARCH ARTICLE

\title{
VELOPHARYNGEAL DYSFUNCTION: A SYSTEMATIC REVIEW OF MAJOR INSTRUMENTAL AND AUDITORY-PERCEPTUAL ASSESSMENTS.
}

\author{
Doaa M. Elkady ${ }^{1}$, Cecile G. Michael ${ }^{2}$ and Ahmed E. Fayyad ${ }^{3}$. \\ 1. Assistant lecturer of Prosthodontics, Faculty of Oral and Dental Medicine, Cairo University. \\ 2. Professor of Prosthodontics, Faculty of Oral and Dental Medicine, Cairo University. \\ 3. Associate Professor of Prosthodontics, Faculty of Oral and Dental Medicine, Cairo University.
}

\section{Manuscript Info}

Manuscript History

Received: 15 October 2017

Final Accepted: 17 November 2017

Published: December 2017

Key words:-

Cleft Palate, hard palate, soft palate; communication disorders and velopharyngeal sphincter.

\begin{abstract}
Inroduction: The main problem in patients with velopharyngeal deficiency is the impairment of speech particularly those with cleft lip, palate, hard and soft palatal defects. Thus, such patients need to undergo both instrumental and auditory perceptual assessments. Objectives: To investigate the most appropriate methods used to evaluate velopharyngeal function in individuals with cleft lip, palate , hard and soft palate defects and to determine whether there is an association between instrumental and auditory-perceptual assessments. Methods: Electronic searching was performed in the following databases, Pubmed (2013 to 2017) and Cochrane (2013 to 2017). In addition, hand searching was performed in maxillofacial and phonetics related journals and through the references of included studies. Summary of findings: Our search of the previously mentioned scientific databases retrieved 35 manuscripts , 30 manuscripts of them remained after full text screening. Then we refined our search in accordance with the objectives of the present review, inclusion and exclusion criteria and found only 2 manuscripts .The hand searching also revealed 2 manuscripts so the included studies from our scientific search was 4 manuscripts that included explanatory description of the best diagnostic method for assessment of velopharyngeal dysfunction.

Conclusion: The included manuscripts revealed a great association between the auditory perceptual assessment results and the instrumental assessment results. So the use of both assessment techniques may be necessary to ensure the reliability of results.
\end{abstract}

Copy Right, IJAR, 2017,. All rights reserved.

\section{Introduction:-}

Velopharyngeal sphincter is a three-dimensional muscular valve formed by the soft palate together with the lateral and posterior pharyngeal walls which are important for intimate velopharyngeal closure.[1]

The complete velopharyngeal closure is required during swallowing and for production of most of speech sounds except for the nasal consonants where the velopharyngeal part remains open to allow for sound transmission into the nasal cavity.[2] Any alteration in the velopharyngeal mechanism resulting from lack of tissues for achievement of

Corresponding Author:- Doaa M. Elkady. 
proper velopharyngeal closure (velopharyngeal insufficiency), lack of neuromuscular competence in the movement of velopharyngeal structures (velopharyngeal incompetence) or as a consequence of mislearning or maladaptive velopharyngeal function not related to physical or neuromuscular problems resulting in velopharyngeal deficiency [3]

One of the consequences of Velopharyngeal insufficiency is airflow escape and hypernasality which can be distinguished by speech and nasal resonance abnormalities, while secondary effects are disorders in speech articulation.[4]

Prosthetic management of velopharyngeal insufficiency is carried out by means of a pharyngeal obturator which is a removable maxillary prosthesis used to restore the soft palatal defect .[5] A pharyngeal obturator is a removable maxillary prosthesis with posterior extension used to restore the soft palatal defect and to separate the nasopharynx and oropharynx during a function to ensure proper velopharyngeal closure.[6]

Several methods for evaluating the velopharyngeal mechanism have been designed. The choice of a specific evaluation method is directly related to the focus of interest of a clinical investigation and its need for accuracy. The use of 1 auditory-perceptual assessment and at least 1 instrumental assessment is recommended for the analysis of velopharyngeal function .[7, 8]

The main method for detecting possible changes in speech nasality is the auditory-perceptual assessment as it's easily performed and provides data on the function of velopharyngeal structures during speech production. So, this evaluation has the ability to detect specific symptoms of cleft palate that may or may not be associated with velopharyngeal deficiency .[9,10,11,12,13,14]

Videonasoendoscopy is also one of the most appropriate tools for assessment of the velopharyngeal deficiency because it shows dynamic, direct, and natural images of the anatomical structures of the nasal cavity, pharynx, and larynx. [15]

Velopharyngeal closure patterns and the presence of a velopharyngeal gap can be detected during performance of the test. It can also identify such patterns during speech production, including the characteristics and degree of movement of the soft palate and pharyngeal walls $[16,17,18]$.

Because of the complexity of the anatomy and physiology of the velopharyngeal mechanism and it's great influence on the speech intelligibility in individuals with cleft lip, hard and soft palate defects. Therefore, the objective of the present review was to investigate the main methods used to evaluate the velopharyngeal function in individuals with cleft lip, hard and soft palate defects, and to determine whether there is an association between instrumental assessment results and auditory-perceptual assessments.

\section{Methods:-}

This systematic review was developed according to PRISMA and it was formulated using the PICO (participant, intervention, comparison and outcome (1) Participant: Patients with velopharyngeal dysfunction. (2) Intervention: videonasoendoscopy(instrumental assessment) (3) Comparison: auditory perceptual assessment. (4) Outcome: hypernasality and velopharyyngeal dysfunction assessment.

The proposed and focused questions were "What are the main methods of velopharyngeal function assessment used in patients with velopharyngeal dysfunction ? Is there an association between videonasoendoscopy results (instrumental assessment) and auditory perceptual assessments results?

Electronic searching was performed in the following databases (1) Pubmed (2013 to 2017) (2) Cochrane (2013 to 2017) and our search included all the manuscripts published between 2013 and 2017.

This period was selected based on the published systematic review on 2013 and the need to update this systematic review and include the articles published in the last five years. First, we selected the keywords to search the databases considering our research questions. The following keywords were used alone and in combination with the other terms: velopharyngeal insufficiency, velopharyngeal incompetence, hard and soft palate defects, instrumental assessment, subjective speech evaluation ,hypernasality assessment and speech disorders assessment. 
Hand searching of the years from 2013 to 2017 was done also in following in maxillofacial and phonetics related journals and through the references of included studies.

\section{Selection criteria:-}

The literature review and data extraction were performed by two authors who worked independently. The full texts of all potentially relevant manuscripts were obtained and analyzed separately by 2 reviewers based on the following inclusion criteria:

\section{The inclusion criteria were:-}

1. Cohort and retrospective studies.

2. Patients with velopharyngeal dysfunction

3. At least 1 instrumental and/or 1 auditory-perceptual assessment for screening of velopharyngeal deficiency.

4. Manuscripts described the methods and criteria used for the analysis of the velopharyngeal functional assessment results.

5. English-language publication.

Finally, 2 researchers who specialize in the area of interest in the present study revised the selection of manuscripts with the purpose of refining the results.

\section{Systematic search flow diagram:-}

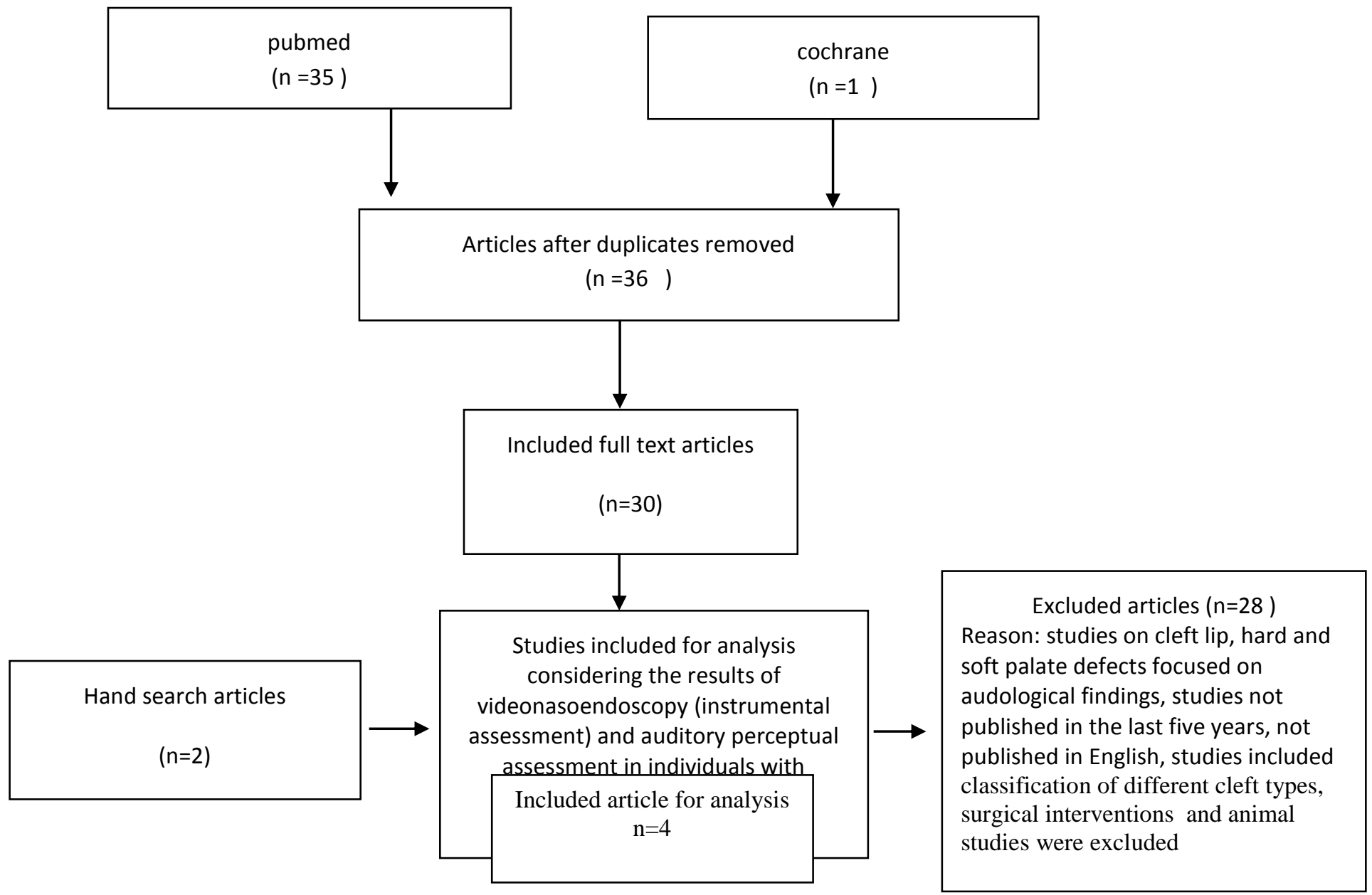

Fig .1:- (search strategy)

However the exclusion criteria were:-

1. Case reports, reviews, non-clinical studies. 
2. Finite element analysis studies.

3. Studies on cleft lip, hard and soft palate defects focused on audiological findings.

4. Classification of different cleft types and surgical interventions were also excluded.

5. Animal studies.

The electronic search was done through 3 steps. Step 1, articles titles was screened from the 2 electronic databases and each investigator independently analyzed relevant titles regarding the selected inclusion criteria., any disagreement was discussed by the 2 authors. At step 2, both authors separately screened the abstracts of all selected titles. Again, any disagreement was discussed by the 2 authors. At step 3, the investigators studied all full text articles that were included. Selection of the article based on the inclusion and exclusion criteria that were applied and the validity of the methodology, and then the qualitative and quantitative data was extracted.

A data extraction form was developed to collect general information (title, year of publication, country, type of defect, age group and tools used in the study as regards direct instrumental assessment, indirect instrumental assessment and clinical evaluation method)

Extraction sheet for the included studies:-

Table I:- Characteristics of the study.

\begin{tabular}{|l|l|l|l|}
\hline Author & Country & Type of defect & Age group \\
\hline Lauren et al.,2013 & Brazil & Cleft lip and palate & Not available \\
\hline Rafalei et al.,2014 & Brazil & Not available & Not available \\
\hline Youssef et al., 2015 & Egypt & Velopharyngeal insufficiency & 4-16 years \\
\hline $\begin{array}{l}\text { Georgievska-Jancheska } \\
\text { et al., }\end{array}$ & Macdonia & Cleft palate/cleft lip and palate & Not available \\
\hline
\end{tabular}

Table 2:- Tools used in the study.

\begin{tabular}{|l|l|l|l|}
\hline Authors & $\begin{array}{l}\text { Direct instrumental } \\
\text { assessment }\end{array}$ & $\begin{array}{l}\text { Indirect instrumental } \\
\text { assessment }\end{array}$ & Clinical evaluation \\
\hline Lauren et al.,2013 & Videonasoendoscopy & Not used & Auditory-perceptual assessment \\
\hline Rafalei et al.,2014 & Not used & Not used & Auditory perceptual assessment \\
\hline Youssef et al., 2015 & $\begin{array}{l}\text { Nasopharyngscopy } \\
\text { and Multiview } \\
\text { videofluroscopy }\end{array}$ & Nasometer & Velopharyngeal gap size \\
\hline $\begin{array}{l}\text { Georgievska- } \\
\text { Jancheska et al.,2016 }\end{array}$ & Not used & Not use & $\begin{array}{l}\text { Perceptual assessment using Czermak } \\
\text { mirror fogging test }\end{array}$ \\
\hline
\end{tabular}

\section{Results and discussion:-}

Considering the objectives of the present review, our search of the previously mentioned scientific databases retrieved 35 manuscripts, 30 manuscripts of them remained after full text screening .Then we refined our search in accordance with the objectives of the present review, inclusion and exclusion criteria and found only 2 manuscripts .The hand searching also revealed 2 manuscripts so the included studies from our scientific search was 4 manuscripts that included explanatory description of the best diagnostic method for assessment of velopharyngeal dysfunction.

Table 1 has shown the characteristics of each study included in this review. The selected studies were performed in different countries including Brazil, Canada, Egypt and Macdonia. These studies included patients with different types of velopharyngeal deficiencies including cleft lip and palate, soft and hard palate defects and submucous cleft. They have a wide range of age, however in most of them the age is not available.

The detailed description of both types of assessment and the parameters used for the analysis of the results of the selected studies are shown in Table 2.

The direct instrumental assessments used in these studies included Videonasoendoscopy, Nasopharyngscopy and Multiview videofluroscopy performed to view the velopharyngeal mechanism. They all perform the same purpose of viewing the velopharyngeal mechanism. The indirect instrumental assessment used in the included studies was the 
nasometer. However the clinical evaluation was performed by auditory perceptual assessment, perceptual assessment using Czermak mirror fogging test and by the aid of speech accessibility rating. The analysis parameters for each type of instrumental assessment and auditory perceptual assessment differed for each study.

The main target to conduct such a systematic review was due to the problem faced by health professionals who provide clinical care to the patients with velopharyngeal deficiencies with subsequent effect on the verbal communication and the speech outcome. It's extremely necessary for the prosthodontics to ensure the intimate contact between the bulb portion of the pharyngeal obturator with the posterior and lateral pharyngeal walls to ensure adequate velopharyngeal function during prosthodontic management of patients with hard and soft palate defects which significantly affect the speech intelligibility. Discrepancies in findings relating to speech outcome as regards the degree of hypernasality and the degree of velopharyngeal closure are common in clinical practice. Because of the complex physiology of the velopharyngeal sphincter and the high risk of biases by the patient or the examiner .So it was highly important to detect the association between the clinical and instrumental evaluation for achieving a clinical conclusion to the real condition of the velopharyngeal mechanism.

Lauren et al.,(2013) reported a retrospective cross sectional study assessing 49 subjects of both sexes with cleft lip and palate ,they compared the findings of auditory perceptual assessment and those of videonasoendoscopy in patients with cleft lip and palate. The results revealed that the subjects with moderate to severe hypernasality had more severe velopharyngeal closure impairment than those with less severe condition. The interaction between hypernasality severity and the presence of other disorders increases the likelihood of having a gap ranging from moderate to large. Although they found discrepancies between the findings of auditory perceptual assessment and instrument based evaluations, they observed an association between the findings of these two evaluation methods.

Rafalei et al.,(2014) carried out two logistic regression models to verify the possibility of predicting the possibility of velopharyngeal closure using the following characteristics: rating of velopharyngeal closure (adequate, border line, inadequate) ,degree of nasality(absent, mild, moderate, severe) and the presence of nasal air emission determined perceptually by three experienced speech language pathologists, In the first model the results revealed that 65 of the 100 samples were rated in the correct velopharyngeal closure category, with 42 adequate and 23 inadequate.

The borderline velopharyngeal closure was not predicted. The second model rated 31 of the 43 samples in the correct category, with 21 adequate VP closure, 5 in the borderline velopharyngeal closure and 5 inadequate. There was no difference between the two models. However, the second model showed a higher proportion of accuracy (7\%) than the first one, and it has also predicted the borderline velopharyngeal closure. They explained that although there is a consensus in the literature that the auditory perceptual assessment of speech is the means through which the speech language pathologist may identify the changes in speech, classifying its severity and thus, defining the conduct and assessing the treatments performed, However, it is a subjective method and, therefore, subject to mistakes and influences of various factors. This may be related to the internal standards of each listener, that is, the individual references of each evaluator that differ from one another. This led clinicians and researchers into searching strategies to enhance the perceptual assessment.

The main change was the use of recordings of speech samples, which allowed the assessing of the speech by more than one listener. From there, several studies showed the importance of the presence of the analysis of speech symptoms by different evaluators and of obtaining agreement among them. Specifically which related to hypernasality, a high level of agreement between evaluators is difficult to be obtained. Although the evaluators consulted had 12 years of experience on speech assessment in the presence of cleft palate.

They concluded that the high level of inter-judge agreement as for the hypernasality degree positively affected the prediction of velopharyngeal closure. This means that, besides the perceptual judgment made by more than one listener, in the auditory-perceptual assessment of the speech characteristics, it is essential to use strategies that ensure high levels of agreement among them, to improve the reliability of results.

Youssef et al.,2015 conducted a prospective correlation study to evaluate the role of auditory perceptual analysis of speech in predicting velopharyngeal gap size. The subjective evaluation of patients' speech was carried out using four-point severity scales. This included degree of hypernasality, audible nasal emission of air, the compensatory articulations (glottal and pharyngeal articulation), and the overall intelligibility of speech. Nasalance scores for oral 
and nasal sentences were measured. A combination of nasopharyngoscopy and multiview videofluoroscopy was used to measure velopharyngeal gap size, which was rated using the scale proposed by Golding-Kushner and colleagues. The studied patients were classified into three groups on the basis of the estimated gap size (small, moderate and large VP gap groups). There was a positive correlation between all studied auditory perceptual analysis variables and VP gap size. The degree of hypernasality and overall speech intelligibility had the strongest predictive values, followed by glottal articulation, nasal emission, pharyngeal articulation, and oral sentence nasalance score. The results of this study indicate that there is a significant and clinically relevant relationship between velopharyngeal gap size and the perceived characteristics of speech as regards degree of hypernasality, nasal emission, glottal articulation, pharyngeal articulation and overall speech intelligibility. Results showed that the size of the gap increases as the severity of speech impairment increases. This finding suggested that the size of the velopharyngeal opening may be predicted, to some extent, on the basis of perceptual assessment of speech. Perceptually predicting the gap size could help the speech-language pathologist to anticipate the appropriate line of intervention without the need for invasive procedures.

Georgievska-Jancheska et al.,2016 performed a study to establish a link between the nasal air escape and the perceptual symptoms in the speech of patients with cleft palate or cleft lip and palate using auditory visual perceptual procedures for determining the influence of velopharyngeal dysfunction on speech. They conducted a two independent auditory-visual perceptual examinations for estimating the velopharyngeal function. The first is the mirror fogging test which is considered the most relevant procedures for assessing speech disorders with cleft palate and velopharyngeal dysfunction. This test for nasal airflow is useful for assessing the function of the velopharyngeal mechanism . In addition to this, auditory-perceptual testing was conducted by means of Pittsburgh Weighted Speech Scale particularly standardized for assessing the velopharyngeal insufficiency and also one of the most commonly used in practice. They concluded that the perceptual speech symptoms and the nasal air escape provide unique insight into the state and role the velopharyngeal sphincter in speech. However among the limitations of this study was that during the intraoral examination the presence of cleft (cleft palate or cleft lip and palate) was established, but not its size as well nor how the size of the cleft affects speech. That is, how it affects the level of nasal emission or perceptual speech symptoms.

\section{Conclusions:-}

In the present systematic review, the included manuscripts revealed a great association between the auditory perceptual assessment and the instrumental assessment for detection of the presence as well as the size of the velopharyngeal gap. However auditory perception assessment is a subjective method and based mainly on the individual variations which necessitate a reliable instrumental assessment to ensure high levels of agreement among them and to improve the reliability of results.

\section{References:-}

1. Hardik K Ram., and Rupal J Shah: A novel approach for velopharyngeal prosthetic rehabilitation:caseseries,International journal of healthcare \& biomedical research;,1:70-76,2013.

2. Johns, D. F., Rohrich, R. J. and Awada, M.: Velopharyngeal incompetence: a guide for clinical evaluation. Plastic and Reconstructive Surgery, 112, 1890-7; quiz 1898,1982. 2003.

3. Trindade, I. E. K., Genaro, K. F., Yamashita, R. P., Miguel, H. C. and Fukushiro, A. P.: Proposal for velopharyngeal function rating in a speech perceptual assessment. Pro-Fono : Revista de Atualizacao Cientifica, 17, 259-262.2006.

4. Abreu A., Levy D., Rodriguez E., and Rivera I.: Oral rehabilitation of a patient with complete unilateral cleft lip and palate using an implant-retained speech-aid prosthesis: Clinical report. Cleft Palate Craniofac. J.;,44:673-677,2007.

5. Shifman, A., Finkelstein, Y., Nachmani, A. and Ophir, D.:Speech-aid prostheses for neurogenic velopharyngeal incompetence. Journal of Prosthetic Dentistry, 83(1), 99-106. 2000.

6. Coskun, A., Yaluğ, S., and Yazicioğlu, H: Fabrication of a meatus obturator on a titanium framework with a 1-step impression. Quintessence International (Berlin, Germany : 1985), 37, 575-578.2006.

7. Rocha DL. Insuficiência Velofaríngea. In: Mélega JM. Cirurgia Plástica: Fundamentos e Arte - Cirurgia reparadora de Cabeça e Pescoço. Rio de Janeiro: Medsi; 2002. p. 178 - 96.

8. Golding-Kushner KJ, et al. Standartization for the reporting of nasopharyngoscopy and multiview videofluoroscopy: a report from an international working group. Cleft Palate J. 1990;27:337-48. 
9. Conley SF, Gosain AK, Marks SM, Larson DL. Identification and assessment of velopharyngeal inadequacy. Am J Otolaryngol. 1997; 18:38-46.

10. Shprintzen RJ. Instrumental Assessment Of Velopharyngeal Valving. In: Shprintzen RJ, Bardach J. Cleft palate speech management: A multidisciplinary approach. St. Louis: Mosby; 1995. p. 221-56.

11. Trindade IEK, Trindade Junior AS. Avaliação funcional da inadequação velofaríngea. In: Carreirão $S$, Lessa S, Zanini AS, editors. Tratamento das fissuras labiopalatinas. 2nd ed. Rio de Janeiro: Editora Revinter; 1996. p. 223-35.

12. Sell D, Harding A, Grunwell P. A screening assessment of cleft palate speech (Great Ormond Street Speech Assesmente). Eur J Disord Commun. 1999;29:1-15.

13. Genaro KF, Yamashita RP, Trindade IKE. Avaliação clínica e instrumental na fissura labiopalatina. In: Ferreira LP, Befi- Lopes DM, Limongi SCO. Tratado de Fonoaudiologia. São Paulo: Rocca; 2004. p. 456-77.

14. Laczi E, Sussman JE, Stathopoulos ET, Huber J. Perceptual evaluation of hypernasality compared to HONC measures: The role of experience. Cleft Palate Craniofac J. 2005;42:202-

15. Pontes PAL, Behlau MS. Nasolaringoscopia. In: Altmann EBC. Fissuras labiopalatinas. 4th ed. Carapicuíba: Pró-fono; 2005. p. 175-83.

16. Kuehn DP, Henne LJ. Speech Evaluation and Treatment for Patients With Cleft Palate. Am J Speech Lang Pathol. 2003;12:103-9.

17. Shprintzen RJ. Nasopharyngoscopy. In: Bzoch KR, editors. Communicative Disorders Related To Cleft Lip And Palate. 5th ed. Boston: Little \& Brown; 2004.

18. Williams WN, Heningsson G, Pegoraro-Krook MI. Radiographic assessment of Velopharyngeal function for speech. In: Bzoch KR, editor. Communicative disorders related to cleft lip and palate. 5th ed. Boston: Little \& Brown; 2004. 